\title{
ORIGINAL
}

\section{ESTUDIO DE LA FERTILIDAD Y VIABILIDAD DE QUISTES HIDATÍDICOS OVINOS}

\author{
José Luis García Llamazares, Ana Isabel Álvarez de Felipe, Pedro Angel Redondo Cardeña y Julio \\ Gabriel Prieto Fernández * \\ Departamento de Fisiología, Farmacología y Toxicología. Universidad de León. \\ * Este trabajo ha sido subvencionado por la Junta de Castilla y León (BOC y L 28-12-93).
}

\section{RESUMEN}

Fundamento: La finalidad de este trabajo es analizar la fertilidad de los quistes hidatídicos de origen ovino, especie de gran interés epidemiológico en la hidatidosis. y la adecuación del gerbillo (Meriones unguiculatus) como modelo experimental para el estudio «in vivo» de dicha hidatidosis, fase preliminar de posteriores estudios terapeúticos.

Métodos: Se ha realizado un estudio de la fertilidad y viabilidad de quistes hidatídicos procedentes de pulmones e hígados de ganado ovino de Castilla y Lcón a través del examen y evaluación de una serie de parámetros entre los que figura la producción de una hidatidosis secundaria experimental en animales de laboratorio.

Resultados: El índice quístico total obtenido fue de 8.57 quistes por ovino inlestado ( 5.97 quistes por pulmón infestado y 5.57 quistes por hígado infestado). El porcentaje de fertilidad obtenido en los quistes hidatídicos de origen ovino que contenían protoescólex viables «in vitro" fue del $43.97 \%(43,02 \%$ en los quistes pulmonares y $46,16 \%$ en los quistes hepáticos). La viabilidad “in vivo» de los protoescólex seleccionados se puso de manifiesto al producirse en el $100 \%$ de los gerbillos inlectados una hidatidosis secundaria experimental.

Conclusiones: Se destaca la validez de los criterios utilizados para estudiar la viabilidad «in vitro» de los protoescólex procedentes de quistes hidatídicos de origen ovino. La hidatidosis secundaria producida en gerbillos nos conduce a considerarlos como especie de experimentación adecuada para la investigación "in vivo" de la hidatidosis de urigen ovino.

Palabras clave: Índice quístico. Fertilidad y viabilidad de quistes hidatídicos. Echinococos granulosus: Gerbillos (Meriones unguticulatus). Zoonosis. Parasitosis.
ABSTRACT

\section{Fertility and viability study of hydatid cysts from ovine}

Background: The purpose of this work is to analyze the fertility of the hydatid cysts from ovine. animal species of great epidemiological interest in the hydatid disease, and the ability of the gerbil (Meriones unguiculatus) as model experimental for the study «in vivo" of this hidatyd disease as preliminary phase of therapentic studies.

Methods: It has been carried out a study of the fertility and viability of hydatid cysts from lungs and livers of ovine from Castilla and Leon by examination and evaluation of parameters among these is the production of a secondary hydatid disease in laboratory animals.

Results: The total cystic index was 8.57 cysts by infested ovine $(5.97$ cysts by infested lung and 5.57 cysts by infested liver). The fertility percentage obtained in hydatid cysts from ovine with "in vitro" viables protoescoleces was $43.97 \%$ being $43.02 \%$ in pulmonary cysts and $46.16 \%$ in hepatic cysts. The viability of protoscoleces was demonstrated by production of a secondary hydatid disease in $100 \%$ of gerbils infested.

Conclusions: It is emphasized the validity of the criteria used to study the viability «in vitro» of the protoescoleces from hydatid cysts of origin ovine. The secondary hydatid produced in gerbils leads us to consider them as experimental animal for investigation «in vivo» of hydatid disease of origin ovine.

Key Words: Cystic Index. Fertility and viability of hydatid cysts. Echinococus granulosus. Gerbils (Meriones unguiculatus).

\section{INTRODUCCIÓN}

Correspondencia

Julio Gabriel Prieto Fernández

Fisiología Animal. Facultad de Veterinaria

Universidad de León. 24071 León. España

Fax: (987) 291267

E-Mail: dffjpf@unileon.es
La hidatidosis, enfermedad producida por la fase larvaria de Echinococcus granulosus y que presenta una prevalencia elevada entre las zoonosis de Castilla y León, es una parasitosis objeto de numerosas investigacioncs. 
Su estudio en el hombre y en los animales hervíboros domésticos (principales hospedadores intermediarios de esta zoonosis), no avanza a la velocidad deseable, debido como ocurre con otras zoonosis, a divcrsas causas: éticas, de dificultad de diagnóstico, del prolongado espacio de tiempo que transcurre entre la infectación y su manifestación, si es el caso, etc.; por ello es necesario recurrir a la investigación sobre otros medios que obvien dichas dificultades y a la vez proporcionen datos que sean lo más fácilmente extrapolables a los hospedadores antes indicados. Por ello, es nuestro objetivo abordar el estudio de la hidatidosis utilizando modelos «in vivo» de animales de experimentación en los que se reproduce la fase larvaria de esta zoonosis mediante la inoculación intraperitoneal de arena y líquido hidatídicos. Son de especial interés para la lucha y erradicación de esta zoonosis las líneas de investigación sobre la quimioterapia de la hidatidosis utilizando los modelos experimentales «in vivo».

Como fase previa a la producción de una hidatidosis secundaria en los animales de experimentación y para la valoración de la efectividad de los fármacos contra el metacéstodo, es necesario evaluar la fertilidad y viabilidad del material hidatídico utilizado como infectante. Dicho material fue obtenido de ovinos procedentes de las provincias de León y Zamora, que además de resultar ser el hospedador intermediario más importante desde el punto de vista epizootiológico, es la especie de ganado de mayor censo en Castilla y León.

\section{MATERIAL Y MÉTODOS}

Se investigaron pulmones e hígados de 30 animales ovinos parasitados (16 tenían infectados únicamente los pulmones y 14 animales tenían infectados los pulmones y el hígado). Los animales, ovino mayor ( $>5$ años) procedentes de León y Zamora, fueron sacrificados en el matadero municipal de León y a continuación las vísceras fueron transportadas al laboratorio a temperatura de refrigeración $\left(+4^{\circ} \mathrm{C}\right)$. El mismo día de su obtención se procede, previa desinfección, a la punción de los quistes hidatídicos para eliminar la presión intraquística y a la apertura de los mismos para la recogida de la arena hidatídica, líquido hidatídico y membrana germinativa. Dicho material infectante fue examinado a fin de comprobar su fertilidad y viabilidad, siguiendo las directrices de la $\mathrm{OMS}^{\perp}$ adaptadas por nuestro grupo de investigación, utilizando como parámetros:

\section{a) Fertilidad de los quistes:}

- morfología del quiste hidatídico y aspecto del líquido hidatídico (fueron seleccionados los quistes hidatídicos con líquido hidatídico transparente y con protoescólex)

b) Viabilidad de los protoescólex «in vitro»:

- morfología de los protoescólex (fueron seleccionados los protoescólex de morfología ovoidea, con ganchos rostelares intactos y con abundantes corpúsculos calcáreos)

- exclusión de tinciones vitales (azul de Tripano $0,32 \%$ ) (fueron seleccionados los quistes con un porcentaje de protoescólex que excluían la tinción, superior al 70\%)

- actividad de células flamígeras o solenocitos.

motilidad y capacidad de evaginación espontáneas de los protoescólex.

Se consideraron viables «in vitro» los protoescólex que, al menos, cumplían los tres primeros criterios señalados en este apartado.

c) Viabilidad de los protoescólex «in vivo»:

- la capacidad infectante mediante inoculación en animales de experimentación

Para provocar una hidatidosis secundaria en animales de experimentación $\mathrm{y}$, de esta 
forma, comprobar la viabilidad de los protoescólex, se elaboraron dosis infectantes de 1.200-1.500 protoescólex mediante su recuento en cámara de McMaster, y utilizando para el lavado, sedimentación y como líquido vehiculizante de los mismos una solución PBS pH 7,2 con $100 \mathrm{UI} / \mathrm{ml}$. de penicilina $G$ sódica y $100 \mu \mathrm{g} / \mathrm{ml}$ de sulfato de dihidroestreptomocina ${ }^{2-4}$. Dicha dosis es inyectada intraperitonealmente en 165 gerbillos (Meriones unguiculatus) previa y posterior desinfección local del punto de inoculación. Dichos animales fueron sacrificados a partir de 3,5 meses postinfectación a fin de comprobar la hidatidosis secundaria experimental. En las mismas condiciones se infectaron 50 ratas (Rattus norvegicus var. Wistar) que fueron sacrificadas a los 8-9 meses.

Se calculó el promedio de quistes hidatídicos por animal y órgano parasitado mediante las fórmulas siguientes:

- Índice Quístico Total $=\mathrm{N} .^{\circ}$ quistes totales / N. ${ }^{\circ}$ de animales parasitados

—Índice Quístico Pulmonar = N. ${ }^{\circ}$ quistes pulmonares / N. ${ }^{\circ}$ pulmones parasitados

- Índice Quístico Hepático $=$ N. ${ }^{\circ}$ quistes hepáticos $/ \mathrm{N}^{\circ}$ hígados parasitados

- Índice Pulmón $/$ Hígado $=$ N ${ }^{\circ}$ pulmones parasitados $/ \mathrm{N} .^{\circ}$ hígados parasitados

\section{RESULTADOS}

En los 30 pulmones y 14 hígados infectados se observaron un total de 257 quistes hidatídicos (179 de localización pulmonar y 78 de localización hepática), obteniéndose los siguientes resultados:

- Índice Quístico Total = 8,57

— Índice Quístico Pulmonar = 5,97

—Índice Quístico Hepático = 5,57

— Índice Pulmón/Hígado = 2,14
Todos los quistes hidatídicos fueron examinados a fin de comprobar su fertilidad y la viabilidad «in vitro» de los protoescólex. De los 257 quistes estudiados, 113 cumplían los criterios de selección antes expuestos, es decir un 43,97\%. En el caso de los quistes de localización pulmonar (77 de 179), el porcentaje fue de un 43,02\%; en los hepáticos (36 de 78), el porcentaje fue de un $46,16 \%$ (tabla 1).

Se comprobó la viabilidad «in vivo» de los protoescólex procedentes de los quistes hidatídicos considerados fértiles y que así mismo cumplían los criterios de selección de viabilidad «in vitro» de los protoescólex, mediante la observación de una hidatidosis secundaria experimental. La infestación experimental se produjo en el $100 \%$ de los 149 gerbillos supervivientes. En la necropsia de las 46 ratas supervivientes infestadas, en ningún caso se observó signo indicativo de hidatidosis secundaria.

\section{DISCUSIÓN}

A pesar del hecho scñalado por Fernán$d e z^{5}$ de que el hígado es el filtro más importante, en la mitad de los ovinos parasitados investigados no se observó infectado dicho órgano (16 de 30). En aquellos en que lo estaba (14 de 30), también se encontraban afectados los pulmones. Los índices quísticos de parasitación pulmonar y hepático fueron similares $(5,97$ y 5,57 , respectivamente).

El tamaño de muestra analizada - 30 animales- es insuficiente para extraer conclusiones extrapolables sobre la localización orgánica preferente de los quistes. Existen estudios en los que se ha detectado mayor frecuencia de localización hepática que pulmonar $^{6,7}$, si bien, también existen otros en los que se señala el predominio de la localización pulmonar ${ }^{8.9}$, aunque en la mayoría de los casos fueron realizados sobre pequeños números de muestras. Ello puede deberse, según Heath ${ }^{10}$, a que la localización final de los quistes hidatídicos depende más de otros 
Tabla 1

Porcentajes de viabilidad «in vitro» de los protoescólex procedentes de quistes hidatídicos examinados en vísceras de ovinos parasitados

\begin{tabular}{|lcccc|}
\hline Órgano & $\begin{array}{c}\text { Vísceras examinadas } \\
\text { infestadas }\end{array}$ & $\begin{array}{c}\text { Quistes hidatidicos } \\
\text { examinados }\end{array}$ & $\begin{array}{c}\text { Quistes hidatidicos } \\
\text { viables }\end{array}$ & $\begin{array}{c}\text { Quistes hidatidicos } \\
\text { no viables }\end{array}$ \\
\hline Pulmones & 30 & $179(100 \%)$ & $77(43,02 \%)$ & $102(56,98 \%)$ \\
Hígados & 14 & $78(100 \%)$ & $36(46,16 \%)$ & $42(53,84 \%)$ \\
Total & 44 & $257(100 \%)$ & $113(43,97 \%)$ & $144(56,03 \%)$ \\
\hline
\end{tabular}

factores, como el tamaño de las oncosferas de E. granulosus en relación con el de las vénulas y vías linfáticas en las vellosidades intestinales del hospedador intermediario.

El valor del índice quístico total, 8,57 , es similar al detectado por García Marin ${ }^{7}$ (Índ $=7,89$ ), y superior al encontrado por Ghorui $^{11}$ (Índ=3,0) e Himonas ${ }^{12}$ (Índ $=1,1$ ).

Las cifras de viabilidad quística total $(43,97 \%)$ son más elevadas que las señaladas por algunos autores en quistes hidatídicos procedentes de ganado bovino $(3,26 \%)^{13}$, lo que unido al hecho de ser la especie ovina la de mayor censo en Castilla y León y la más afectada por la hidatidosis, contribuye a resaltar la importancia de esta especie en relación con la bovina en el mantenimiento del ciclo de Echinococcus granulosus. Asimismo, este valor es bastante superior al detectado por otros autores en ganado ovino $(23,84 \%)^{7}$.

El porcentaje de viabilidad de los quistes hepáticos $(46,16 \%)$ fue ligeramente superior al de los pulmonares $(43,02 \%)$. La bibliografía señala al respecto una mayor viabilidad en los quistes pulmonares que en los hepáticos ${ }^{7.12}$, aduciéndose entre otras razones que el pulmón ofrece una menor reacción orgánica de cuerpo extraño que el hígado. Es posible que la mayor viabilidad de los quistes hepáticos aquí detectada se deba a que el número de quistes hepáticos muestreados no sea representativo (78 hepáticos frente a 179 pulmonares).
Asimismo, se observó que en todos los gerbillos supervivientes se desarrolló una hidatidosis secundaria, necesitándose menor plazo de tiempo entre la infectación y la observación de los quistes hidatídicos fértiles que el requerido en ratones ${ }^{2}$, mientras que en ningún caso se observó en la rata. Estos datos coinciden con los obtenidos por Thompson $^{14}$ al lograr producir hidatidosis secundaria en gerbillo y no en rata a partir de protoescólex de origen equino, mientras que Heath ${ }^{15}$ tampoco logró infectar ratas con material parasitario de origen ovino.

A la vista de estos resultados puede concluirse que los criterios empleados para el análisis de la viabilidad «in vitro» de los protoescólex son válidos y que el gerbillo es un buen modelo experimental para el estudio «in vivo» de la hidatidosis de origen ovino con fines terapeúticos.

El interés de la investigación sobre esta zoonosis es claro, por lo que todo apoyo al respecto debe ser potenciado a fin de conseguir conocer los factores determinantes de la fertilidad y viabilidad de los quistes hidatídicos y los mecanismos para transformarlos en infértiles y destruirlos, lo que nos llevaría a la erradicación de la Hidatidosis.

\section{AGRADECIMIENTOS}

Los autores agradecen la colaboración prestada por los Veterinarios Oficiales del Matadero Municipal de León en la obtención del material parasitado de origen ovino. 


\section{BIBLIOGRAFÍA}

1. World Health Organisation FAO/UNEP/WHO Guidelines for surveillance prevention and control of echinococcosis/hydatidosis. J. Eckert, M.A. Gemmell and E.J.L. Soulsby (eds). Ginebra: Organización Mundial de la Salud; 1981.

2. Schwabe CW, Kilejian A, Lainas G. The propagation of secondary cysts of Echinococcus granulosus in the mongolian jird, Meriones unguiculatus. J Parasitol 1970; 56: 80-83.

3. Casado N, Criado A, De Armas C, Jiménez A, Rrodríguez-Caabeiro $F$. Estudio del potencial biológico de quistes hidatídicos multivesiculares de origen humano. Rev Ibér Parasitol 1990; 50 : 259-265.

4. García Llamazares JL, Álvarez de Felipe AI, Redondo Cardeña P, Voces Alonso JA, Prieto Fernández JG. In vivo inhibition of the regenerative capacity of hydatid material after treatment with netobimin. Parasitol Res 1997; 83: 105-108.

5. Fernández Sanz S. Estado actual de la quimioterapia en la hidatidosis humana. Libro de ponencias de la IX Reunión Científica Nacional de la Asociación Española de Hidatidología; Diciembre 1990: Mérida, España. (Hidatidología, Libro VI). Madrid; 1991. p. 377-383.

6. Gusbi AM, Awan MAQ, Beesley WN. Echinococcosis in Lybya. II. Prevalence of hydatidosis (Echinococcus granulosus) in sheep. Ann Trop Med Parasitol 1987; 81: 35-41.

7. García Marín JF, Jiménez Palacios S, Peris Palau B, Badiola Díez JJ, Ibiricu Blazquiz A. Estudio epizootiológico de la hidatidosis ovina en La Rio- ja: prevalencia y formas de presentación. Med Vet 1990; 7:163-172.

8. Hayat B, Iqbal Z, Hayat CS, Khan MZ. Incidence and pathology of lungs and livers affected with hydatidosis in sheep. Pak Vet J 1986; 6: 8-10.

9. Pillai KJ, Narayana Rao PL, Surya Rao K. A study on the prevalence of hydatidosis in sheep and goats at Trinipati municipal slaughterhouse. Ind $\mathbf{J}$ Pub Health 1986; 30: 160-165.

10. Heath DD. The migration of oncospheres of Taenia pisiformis, T. serialis and Echinococcus granulosus within the intermediate host. Int $\mathbf{J}$ Parasitol 1971; 1: 145-152.

11. Ghorui SK, Sahai BN. Studies on the incidence of hydatid disease in rumiants. Ind J An Health 1989; 28: 39-41.

12. Himonas C, Antoniadou K, Papadopoulos E. Hydatidosis of food animals in greece: prevalence of cysts containing viable protoscoleces. J Helminthol 1994; 68: 311-313.

13. Moreno Montañez T, Linares F, Martínez Gómez F, Navarrete I. El ganado bovino en la epidemiología de la hidatidosis en la provincia de Córdoba. Libro de ponencias de la III Reunión Científica Nacional de la Asociación Española de Hidatidología; Burgos; 1982. p. 89-91.

14. Thompson RCA. The mongolian gerbil (Meriones unguiculatus) as a laboratory host the cystic stage of Echinococcus granulosus of british horse origin. Int J Parasitol 1976; 6: 505-511.

15. Heath DD. The development of Echinococcus granulosus larvae in laboratory animals. Parasitol 1970; 60: 449-456. 